\title{
Investigation of singularities inherent to Mueller matrix images of biological crystals: diagnostics of their birefringent structure
}

\author{
I.Z. Misevitch, Yu.O. Ushenko, O.G. Pridiy, A.V. Motrich, Yu.Ya. Tomka, O.V. Dubolazov \\ Chernivtsi National University, Optics and Spectroscopy Dept., Chernivtsi 58000, Ukraine
}

\begin{abstract}
In this work, we have theoretically grounded conceptions of singularities observed in coordinate distributions of Mueller matrix elements for a network of human tissue biological crystals. Found is interrelation between polarization singularities of laser images inherent to these biological crystals and characteristic values of above matrix elements. We have determined criteria for statistical diagnostics of pathological changes in the birefringent structure of biological crystal network by using myometrium tissue as an example.
\end{abstract}

Keywords: polarization, Mueller matrix, singularity, biological crystal, birefringence, statistical moment.

Manuscript received 28.05.09; accepted for publication 10.09.09; published online 30.10.09.

\section{Introduction}

In recent years, in laser diagnostics of biological tissue (BT) structures they effectively use the model approach [1] that allows considering this object as containing two components: amorphous and optically anisotropic ones. Topicality of this modeling is related with the possibility to apply the Mueller matrix analysis of changes in polarization properties caused by transformation of the optic-and-geometric structure of anisotropic components in these biological objects [2-7], optical properties of which are often described using the Mueller matrix [8].

Being based on the approximation of a single light scattering, they found interrelation between the set of statistic distribution moments of the $1^{\text {st }}$ to $4^{\text {th }}$ orders $Z^{(j=1,2,3,4)}$ that characterizes orientation and phase structure of BT birefringent architectonics as well as the set of respective moments [9] for two-dimensional distributions of Mueller matrix elements or Muellermatrix images (MMI) [10-14]. In parallel with traditional statistical investigations, formed in recent 10 to 15 years is the new optical approach to describe a structure of polarizationally inhomogeneous fields in the case of scattered coherent radiation. The main feature of this approach is the analysis of definite polarization states to determine the whole structure of coordinate distributions for azimuths and ellipticities of polarization. The so-called polarization singularities are commonly used as these states [15-24]:

states with linear polarization when the direction of rotation for the electric field vector is indefinite, the so-called S-points;
- circularly-polarized states when the azimuth of polarization $(\alpha)$ for the electric field vector is indefinite, the so-called C-points.

Investigations of polarizationally inhomogeneous object fields for BT with different morphology [25 - 27] allowed to ascertain that they possess a developed network of S- and C-points. For example in [26], the authors found interrelations between conditions providing formation of polarization singular points and particularity of the orientation-phase structure of biological crystals present in territorial matrix of human tissue architectonic network. These interrelations served as a base to make statistical and fractal analyses of distribution densities for the number of singular points in BT images. As a result, the authors confirmed the efficiency of this method for investigation of object fields to differentiate optical properties of BT with a different morphological structure and physiological state.

It should be noted that studied in the works $[26,27]$ were only statistical sets of amounts of S- and C-points for polarization inhomogeneous object fields. In these cases, information about the structure of topological distributions inherent to polarization states around these points was outside researchers' attention. Besides, the mechanisms of direct formation of polarization singularities in biological crystals as well as interferential formation of these polarization states due to a multiple scattering were distinguished not so clearly.

Starting from it, a further analysis of mechanisms and scenarios providing formation of polarization singularities in the field of laser radiation scattered by a biological object seems actual. 


\section{Interrelation between the Mueller matrix and polarization images of biological tissues}

As a mathematical apparatus to describe mechanisms of polarization singularity formation by a biological object, one can use the Mueller matrix that entirely describes its optical properties

$$
\{F\}=\left\|\begin{array}{llll}
f_{11} & f_{12} & f_{13} & f_{14} \\
f_{21} & f_{22} & f_{23} & f_{24} \\
f_{31} & f_{32} & f_{33} & f_{34} \\
f_{41} & f_{42} & f_{43} & f_{44}
\end{array}\right\| .
$$

As known from [14], any " $i k$ " element of the Mueller matrix characterizes the degree of transformation taking place with the Stokes vector parameter $U^{0}{ }_{k}$ of the probing beam when transforming it into the Stokes vector parameter $U_{i}$ of the object beam

$$
\left\{\begin{array}{l}
U_{2}=f_{22} U_{2}^{0}+f_{23} U_{3}^{0}+f_{24} U_{4}^{0} \\
U_{3}=f_{32} U_{2}^{0}+f_{33} U_{3}^{0}+f_{34} U_{4}^{0} \\
U_{4}=f_{42} U_{2}^{0}+f_{43} U_{3}^{0}+f_{44} U_{4}^{0}
\end{array}\right.
$$

Originating from (2), let us understand as singularities of Mueller matrix elements for biological objects the following "uncertainties"

$f_{i k}=0$.

From the mathematical viewpoint, the relations (3) mean that the mechanisms of transformation of the Stokes vector parameter $U^{0}{ }_{k}$ into $U_{i}$ are indeterminate. In other words, indeterminate is the scenario for transformation of the laser radiation polarization state by biological tissue.

Another singular "state" is described by the following values of Mueller matrix elements

$f_{i k}= \pm 1$.

From the physical viewpoint, the condition (4) means that optical properties of this medium (isotropic, anisotropic, etc.) and the respective mechanisms of transformation of laser radiation polarization states are indeterminate.

Some characteristic examples of optical property singularities are demonstrated by biological tissues that are combinations of isotropic $\{A\}$ and anisotropic $\{F\}$ structures [15]. Each of these components is characterized by intrinsic matrix operators

$$
\{A\}=\left\|\begin{array}{llll}
1 & 0 & 0 & 0 \\
0 & 1 & 0 & 0 \\
0 & 0 & 1 & 0 \\
0 & 0 & 0 & 1
\end{array}\right\| \cdot e^{-\tau l},
$$

where $\tau$ is the extinction coefficient inherent to the layer of biological tissue with the geometric thickness $l$

$$
\begin{gathered}
\{F\}=\| \begin{array}{ccc}
1 & 0 \\
0 & \cos ^{2} 2 \rho+\sin ^{2} 2 \rho \cdot \cos \delta \\
0 & \cos 2 \rho \sin 2 \rho(1-\cos \delta)
\end{array} 0 \\
0 \quad \begin{array}{cc}
-\sin 2 \rho \sin \delta \\
0 & 0 \\
\cos 2 \rho \sin 2 \rho(1-\cos \delta) & \sin 2 \rho \sin \delta \\
\sin ^{2} 2 \rho+\cos ^{2} 2 \rho \cos \delta & \cos 2 \rho \sin \delta \\
-\cos 2 \rho \sin \delta & \cos \delta
\end{array} \| .
\end{gathered}
$$

Here, $\rho$ is the orientation of an protein fibril in the architectonic network, the matter of which introduces the phase shift $\delta$ between orthogonal components of the laser wave amplitudes.

A comparative analysis of matrixes (5) and (6) shows that the values of elements $a_{i k}$ and $f_{i k}$ coincide in definite conditions

$$
\left\{\begin{array}{l}
a_{i k}=f_{i k}=0 \\
a_{i k}=f_{i k}=1 .
\end{array}\right.
$$

It follows from (7) that it is impossible to separate or differentiate properties of isotropic and anisotropic optical components for biological objects in the points where Mueller matrix elements acquire singular values.

Analysis of information about conditions providing formation of singular values for Mueller matrix elements describing biological tissues (Table 1) allows to predict scenarios for formation of "field" polarization singularities by them and to determine:

- a whole set of positive and negative C-points $(\delta= \pm \pi / 2)$ in the image of biological object, the coordinate position of which is corresponded with the following conditions $f_{44}=f_{22}=f_{33}=0$;

- $\quad$ a whole set of S-points $(\delta=0)$ for a polarization image with arbitrary azimuths $(0 \leq \rho \leq \pi)$ which are corresponded with the conditions $f_{22}=f_{33}=1$. Besides, it is possible to select different types of polarization singularities in the image of biological tissue:

- dextrogyrate $(\delta=+\pi / 2)-(+C)$ and laevogyrate

$(\delta=-\pi / 2)-(-\mathrm{C})$ singular points

$\left\{\begin{array}{c}f_{24,34}=1-"+C " \\ f_{24,34}=-1-"-C "\end{array}\right.$

- "orthogonal" S-points, formation of which is related with orthogonal orientations of the optical axis in biological crystal

$$
\left\{\begin{array}{llll}
f_{34}=0, & S_{45,-45}-\text { point } s & \text { for } & \rho= \pm \frac{\pi}{4} \\
f_{34}= \pm 1, & S_{0,90}-\text { point } s & \text { for } & \rho=0 ; \frac{\pi}{2}
\end{array} .\right.
$$


So, after determining the coordinate distributions of the Mueller matrix for any biological tissue, one can predict a further scenario of formation of polarization singularities in the object field, which set its coordinate topological structure in the form of an aggregate of $\mathrm{S}$ contours [5-10]. Thereof, the next step was to study interrelations between topological singular structures for polarization images of $\mathrm{BT}$ and respective topological structures of two-dimensional distributions for elements of their Mueller matrixes.

Summarized in Table 1 are the data upon relations of Mueller matrix element singular values for BT and respective values of optical axis orientations as well as phase shifts of the anisotropic components for biological crystals.

Table 1. Conditions providing formation of singular values for matrix elements $f_{i k}$

\begin{tabular}{|c|c|c|c|}
\hline \multicolumn{2}{|c|}{$f_{i k}$} & $\rho$ & $\delta$ \\
\hline \multirow{3}{*}{$f_{22}$} & \multirow{2}{*}{0} & all & $\pm \pi / 2$ \\
\hline & & $\pm \pi / 4$ & all \\
\hline & \pm 1 & all & 0 \\
\hline \multirow{3}{*}{$f_{22}=f_{32}$} & \multirow{2}{*}{0} & all & 0 \\
\hline & & $\pm \pi / 4$ & all \\
\hline & \pm 1 & all & 0 \\
\hline \multirow{4}{*}{$f_{24}=f_{42}$} & \multirow{2}{*}{0} & all & 0 \\
\hline & & 0 & all \\
\hline & 1 & $+\pi / 4$ & $+\pi / 2$ \\
\hline & -1 & $-\pi / 4$ & $-\pi / 2$ \\
\hline \multirow{3}{*}{$f_{33}$} & \multirow{2}{*}{0} & all & $\pm \pi / 2$ \\
\hline & & $\pm \pi / 2$ & all \\
\hline & \pm 1 & all & 0 \\
\hline \multirow{4}{*}{$f_{34}=f_{43}$} & \multirow{2}{*}{0} & all & 0 \\
\hline & & $\pm \pi / 4$ & all \\
\hline & 1 & 0 & $+\pi / 2$ \\
\hline & -1 & $-\pi / 2$ & $-\pi / 2$ \\
\hline \multirow{3}{*}{$f_{44}$} & 0 & all & $\pm \pi / 2$ \\
\hline & 1 & all & 0 \\
\hline & -1 & all & $\pi$ \\
\hline
\end{tabular}

Our analysis of the data summarized in Table 1 shows that, among mechanisms providing formation of singular values for Mueller matrix elements by a system of optical single-axis birefringent fibrils in the architectonic network of biological tissue, one can separate two groups that correspond to formation of circular polarization $(\delta= \pm \pi / 2)$ :

- "phase" ones, when singular values of the element $f_{44}=0$ are formed only due to phase shifts $\delta= \pm \pi / 2$ independently of directions inherent to optical axes $\rho$ of biological crystals;

- "orientational" ones, when singular values of the elements $f_{24}=-f_{42}= \pm 1$ are formed due to phase shifts $\delta= \pm \pi / 2$ in dependence on two directions $\rho= \pm \pi / 4$ inherent to optical axes $\rho$ of biological crystals or singular values of the elements $f_{34}=-f_{43}= \pm 1$ are formed due to phase shifts $\delta= \pm \pi / 2$ in dependence on two directions $\rho=0 ; \pi / 2$ inherent to optical axes of biological crystals.

\section{The scheme of experimental studying and method of measuring the singularities in Mueller matrix images of biological tissues}

Fig. 1 shows the traditional optical scheme of a polarimeter to measure sets of MMI for mounts of BT [14].

Illumination was performed with a parallel $(\varnothing=$ $\left.10^{4} \mu \mathrm{m}\right)$ beam of a He-Ne laser $(\lambda=0.6328 \mu \mathrm{m}$, $\mathrm{W}=5.0 \mathrm{~mW})$. The polarization illuminator consists of the quarter-wave plates 3 and 5 as well as polarizer 4, which provides formation of a laser beam with an arbitrary azimuth $0^{0} \leq \alpha_{0} \leq 180^{\circ}$ or ellipticity $0^{0} \leq \beta_{0} \leq 90^{0}$ of polarization.

Polarization images of BT were projected using the micro-objective 7 into the light-sensitive plane (800x600 pixels) of CCD-camera 10 that provided measurements of BT structural elements within the range 2 to $2,000 \mu \mathrm{m}$.

Experimental conditions were chosen in such a manner that spatial-anglular filtration was practically eliminated when forming BT images. It was provided by matching the angular characteristics of light scattering indicatrixes by BT samples $\left(\Omega \approx 16^{0}\right)$ and angular aperture of the micro-objective $\left(\Delta \omega=20^{0}\right)$. Here, $\Omega$ is the angular cone of an indicatrix where $98 \%$ of the total scattered radiation energy is concentrated.

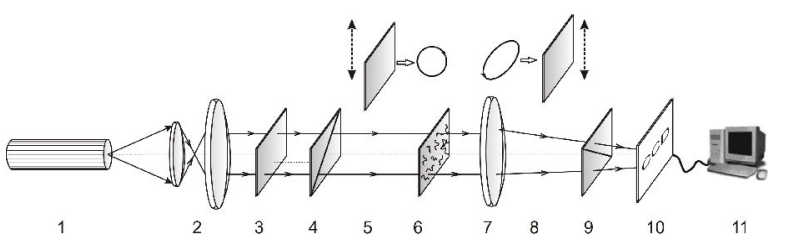

Fig. 1. Optical scheme of a polarimeter: $1-\mathrm{He}-\mathrm{Ne}$ laser; $2-$ collimator; 3 -stationary quarter-wave plate; $5,8-$ mechanically movable quarter-wave plates; 4,9 - polarizer and analyzer, respectively; 6 -studied object; 7 - microobjective; 10 - CCD camera; 11 - personal computer. 
Analysis of BT images was made using the polarizer 9 and quarter-wave plate 8 . As a result, we determined the Stokes vectors for BT images $\left\{S_{j=1,2,3,4}\right\}$ and calculated the ensemble of Mueller matrix elements in one point illuminated with a laser beam (with the cross-section $S=\pi r^{2}$ ) in accord with the following algorithm

$$
\begin{aligned}
& M_{i 1}=0.5\left[S_{i}^{(1)}+S_{i}^{(2)}\right] \\
& M_{i 2}=0.5\left[S_{i}^{(1)}-S_{i}^{(2)}\right] \\
& M_{i 3}=S_{i}^{(3)}-M_{i 1} ; \\
& M_{i 4}=S_{i}^{(4)}-M_{i 1}, i=1,2,3,4 .
\end{aligned}
$$

The indexes 1 to 4 corresponds to the following polarization states of the beam illuminating BT: $1-0^{0} ; 2$ $-90^{\circ} ; 3-+45^{\circ} ; 4-\otimes$ (right circulation).
The method to measure MMI singularities for BT samples was as follows:

- BT mount was illuminated with a laser beam, within the area of which in accord with the algorithm (10) we determined the array $(m \times n=800 \times 600)$ of values for each element of the Mueller matrix

$$
M_{i k}(m \times n)=\left(\begin{array}{c}
M_{i k}^{11}, \ldots \ldots ., M_{i k}^{1 m} \\
M_{i k}^{n 1}, \ldots \ldots \ldots, M_{i k}^{n m}
\end{array}\right)_{l=1,2, \ldots 30} .
$$

- determined for each massif $M_{i k}(m \times n)_{j}$ were coordinate distributions of its singular values.

As an object of the experimental study, we used tissues of a woman matrix (myometrium).

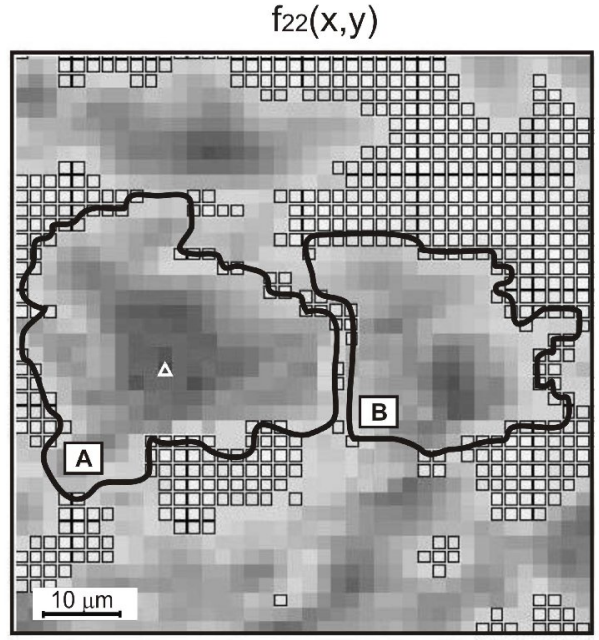

(a)

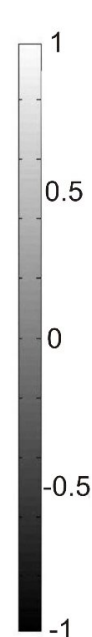

0.5

0.5

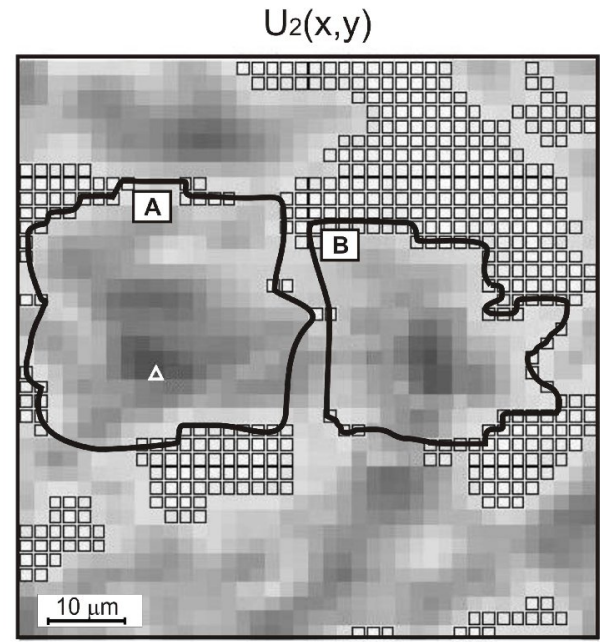

(b)

Fig. 2. Topological structure characterizing the two-dimensional distributions of the matrix element $f_{22}$ for myometrium (a) and $U_{2}$ parameter of the Stokes vector (b) describing its polarization image.

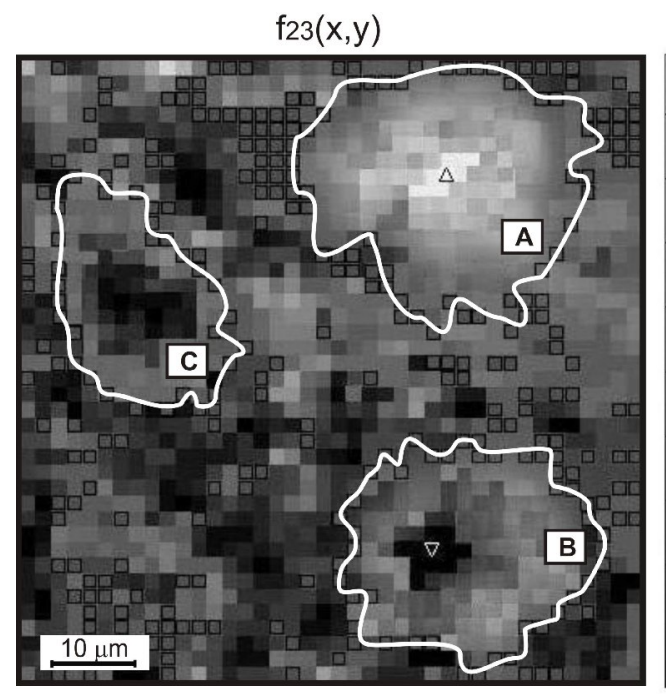

(a)

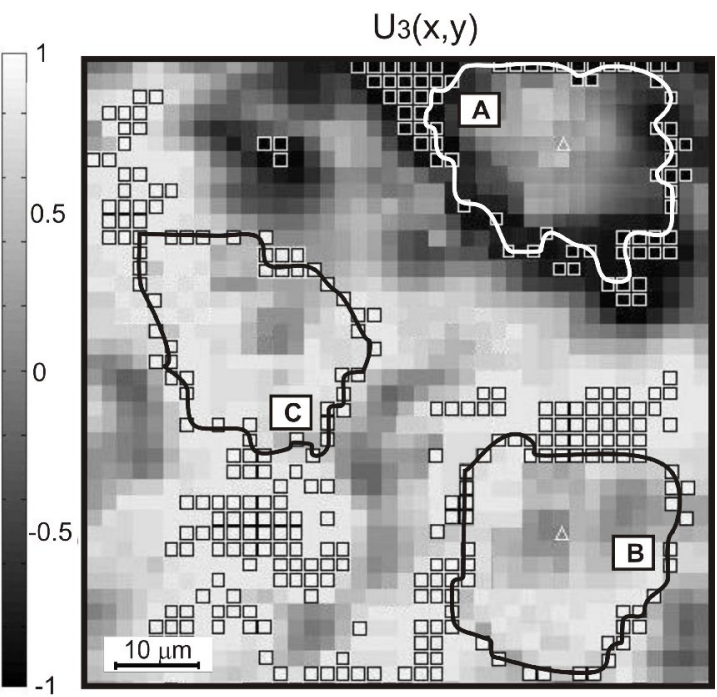

(b)

Fig. 3. Topological structure corresponding to the two-dimensional distributions of the matrix element $f_{23}$ for myometrium layer (a) and $U_{3}$ parameter of the Stokes vector (b) describing its polarization image. 


\section{Local topological structure of experimental Mueller-matrix and polarization images of biological tissues}

In the series of Figs 2 to 5, we show the results of experimental MMI measurements for $f_{i k}$ measurements (fragments $a$ and $b$ ) as well as Stokes-polarimetric study of image topological structure $U_{j}$ (fragment $b$ ) inherent to human conjunctive tissue.

It can be easily seen that the elements $f_{22}$ and $f_{23}$ of the Mueller matrix as well as respective coordinate distributions of $U_{2}$ and $U_{3}$ parameters of the Stokes vector for myometrium polarization image are characterized with closed matrix S-contours in the form of a set of S- and \pm C-lines (Fig. 2, fragments $\mathrm{A}$ and B; Fig. 3, fragments A, B and C).

While for the elements $f_{22}$ and $f_{23}$ of the Mueller matrix as well as respective coordinate distributions of $U_{2}$ and $U_{3}$ parameters of the Stokes vector for myometrium polarization image are characterized with open matrix $\mathrm{S}$-contours in the form of a set of S- and \pm C-lines (Figs 4 and 5, fragments A, B and $\mathrm{C}$ ).

It should be noted that for all the types of topological distributions for MMI of myometrium we

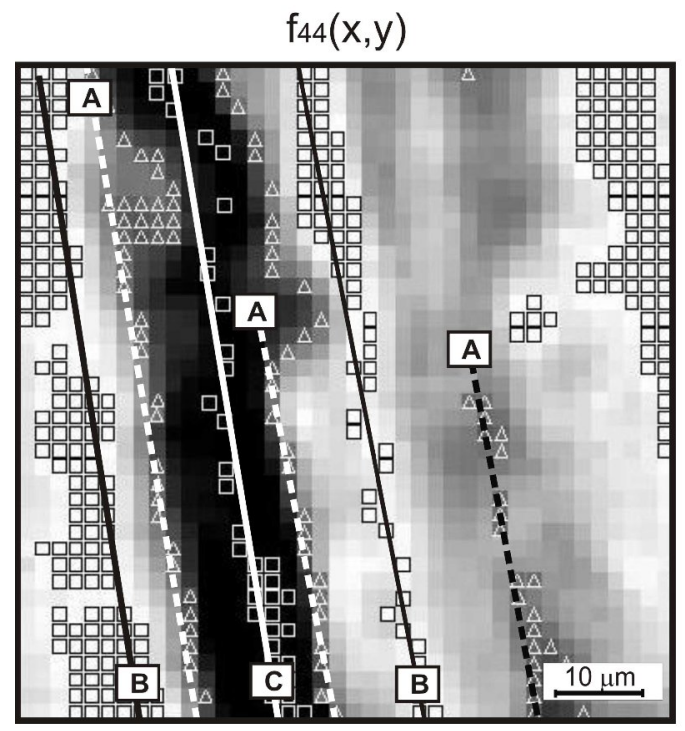

(a)

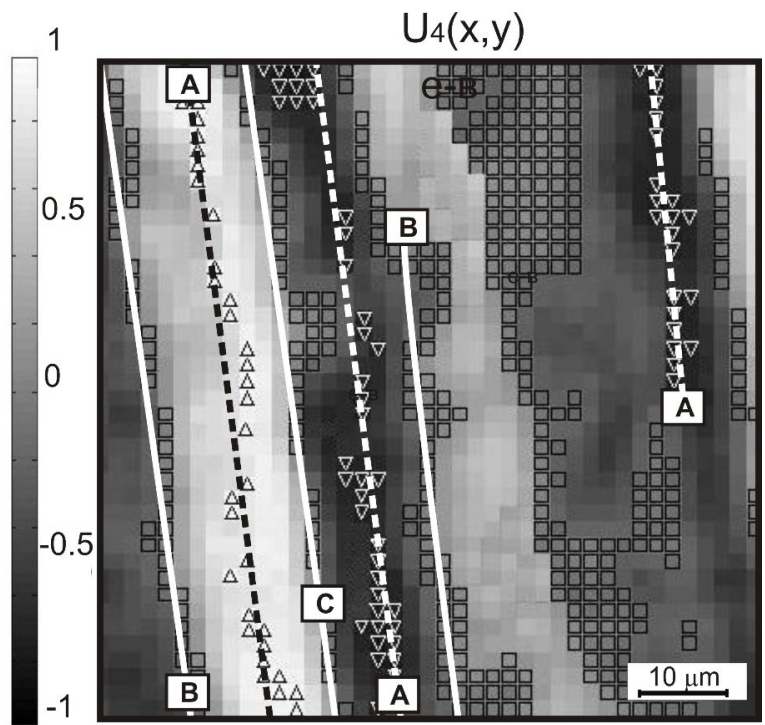

(b)

Fig. 4. Topological structure characterizing the two-dimensional distributions of the matrix element $f_{44}$ for myometrium $(a)$ and $U_{4}$ parameter of the Stokes vector (b) describing its polarization image.

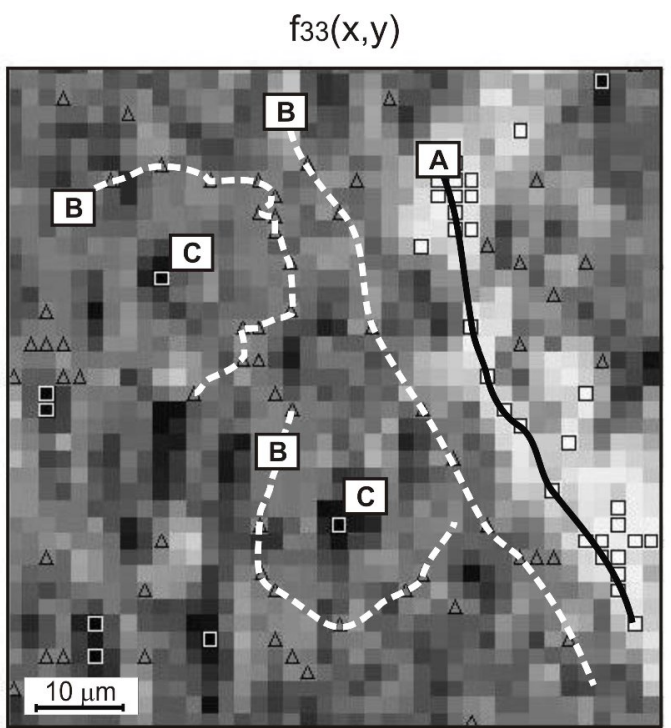

(a)

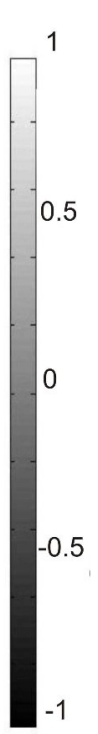

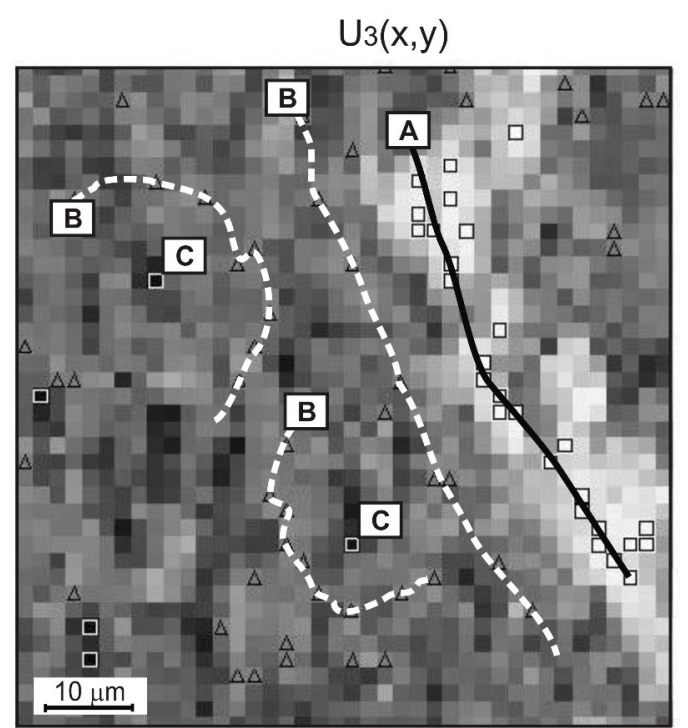

(b)

Fig. 5. Topological structure characterizing the two-dimensional distributions of the matrix element $f_{33}$ for myometrium (a) and $U_{3}$ parameter of the Stokes vector (b) describing its polarization image. 
have revealed one universal regularity. Namely, geometrical places of singular points belonging to one type are necessarily separated with geometrical places for singular points of another type. For instance,

$$
\begin{aligned}
& \left\{f_{22,33,44}(x, y)=1\right\}^{0<f_{i k}<1}\left\{f_{22,33,44}(x, y)=0\right\}^{0<f_{i k}<1} \Leftrightarrow \\
& 0<f_{i k}<1 \\
& \left.\Leftrightarrow \Leftrightarrow f_{22,33,44}(x, y)=1\right\} ; \\
& \left\{f_{34,43,24,42}(x, y)=1\right\}^{0<f_{i k}<1} \Leftrightarrow\left\{f_{34,43,24,42}(x, y)=0\right\}^{0<f_{i k}<1} \Leftrightarrow \\
& 0<f_{i k}<1 \\
& \quad \Leftrightarrow\left\{f_{34,43,24,42}(x, y)=1\right\} ;
\end{aligned}
$$

$$
\begin{aligned}
& \left\{f_{23,32}(x, y)=1\right\}^{0<f_{i k}<1}\left\{f_{23,32}(x, y)=0\right\}^{0<f_{i k}<1} \\
& 0<f_{i k}<1 \\
& \Leftrightarrow\left\{f_{23,32}(x, y)=1\right\} .
\end{aligned}
$$

The expressions (12) to (14) are mathematical equivalents of S-contours in coordinate distributions of the whole set of matrix elements $f_{i k}$ that characterize birefringence of this biological tissue.

Juxtaposition of topological distributions $f_{i k}(x, y)$ and $U_{j}(x, y)$ allows to find a coordinate correlation not only for their singular values but for respective types of "object" and "field" S-contours, too.

Thus, the topological structure of MMI for the ensemble of elements $f_{i k}(x, y)$ that characterizes optical

\section{$\mathrm{f}_{22}(\mathrm{x}, \mathrm{y})$}

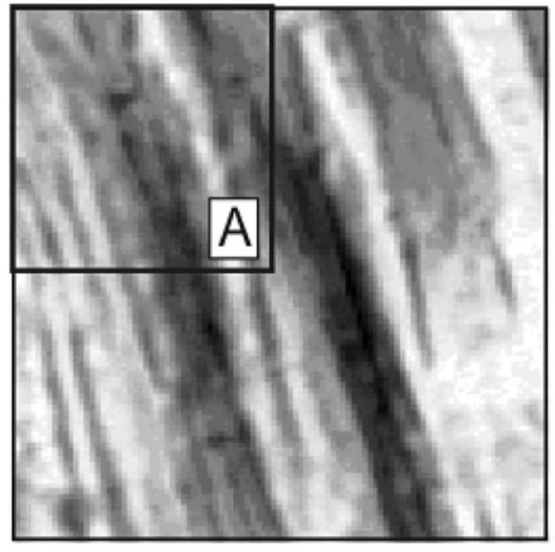

(a)

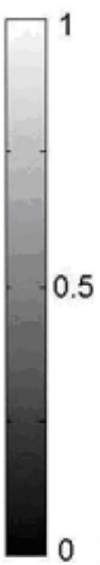

0.5

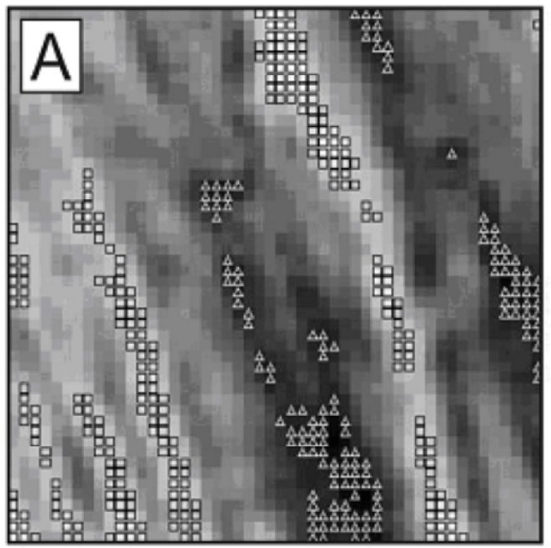

(b)

Fig. 6. Coordinate structure of the matrix element $f_{22}$ for myometrium (a). A - coordinate distribution of singular values (b) S-points $\left(f_{22}=1\right)$ labeled as $(\square)$ and + C-points $\left(f_{22}=0\right)$ labeled as $(\Delta)$.

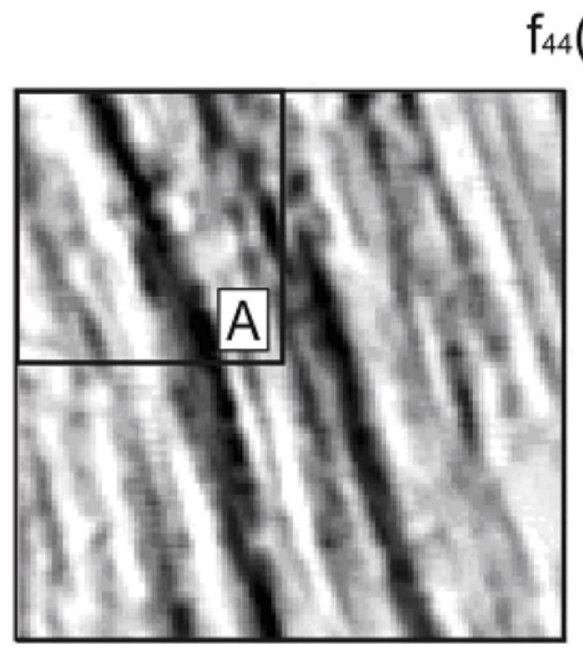

(a) $f_{44}(x, y)$

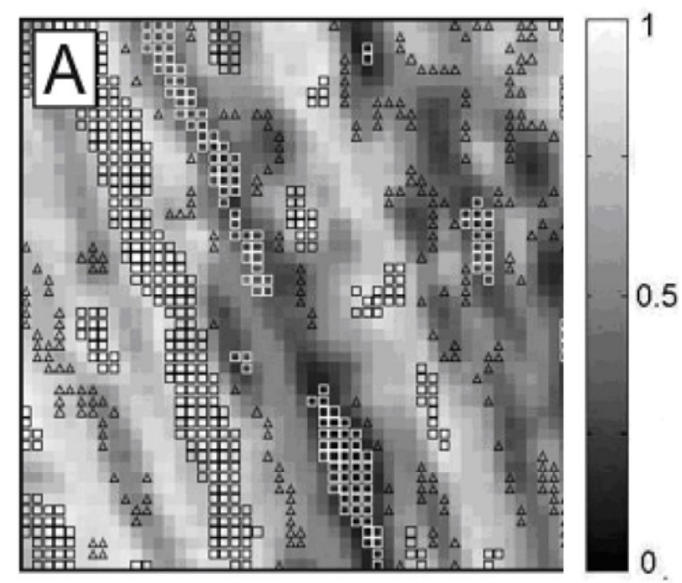

(b)

Fig. 7. Coordinate structure of the matrix element $f_{44}$ for myometrium (a). A - coordinate distribution of singular values (b) S-points $\left(f_{44}=1\right)$ labeled as $(\square)$ and + C-points $\left(f_{44}=0\right)$ labeled as $(\Delta)$. 


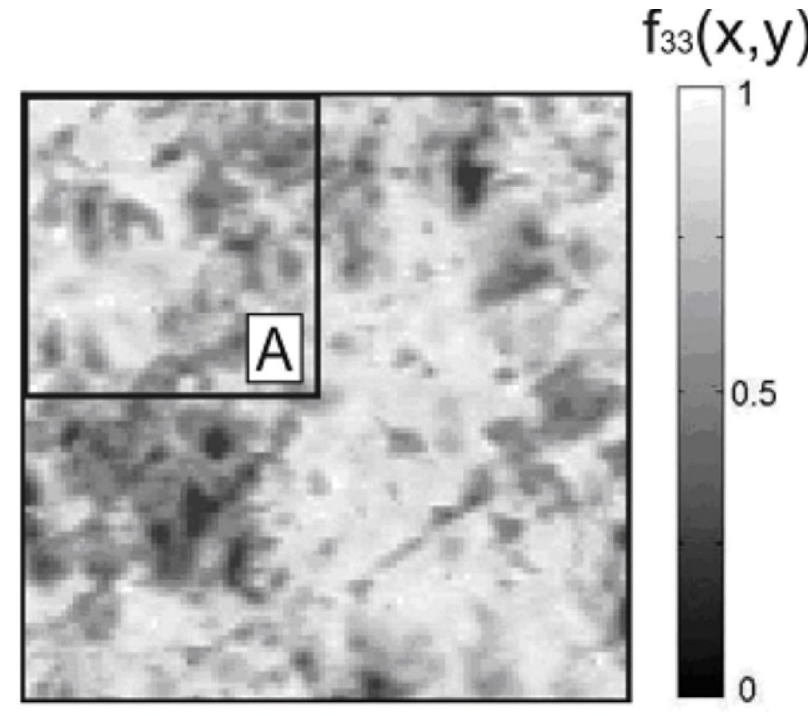

(a)

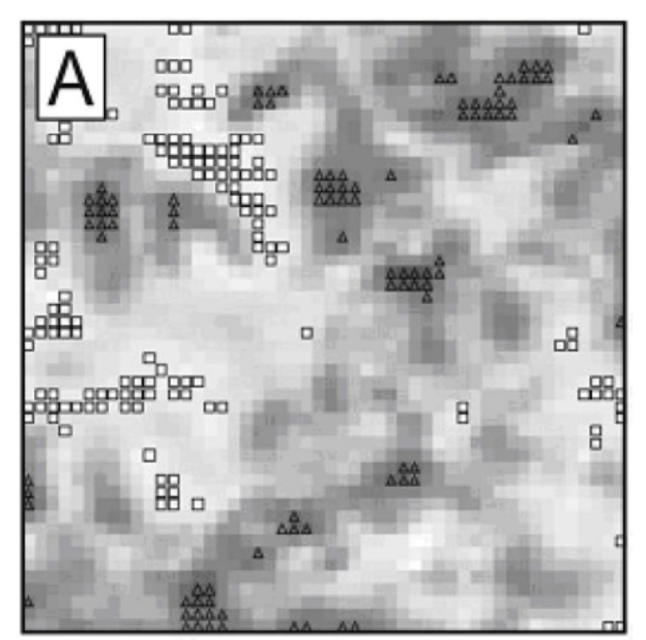

(b)

Fig. 8. Structure of the matrix element $f_{33}$ for myometrium (a). A - coordinate distribution of singular values (b) - S-points $\left(f_{33}=1\right)$ labeled as $(\square)$ and $+\mathrm{C}$-points $\left(f_{33}=0\right)$ labeled as $(\Delta)$.

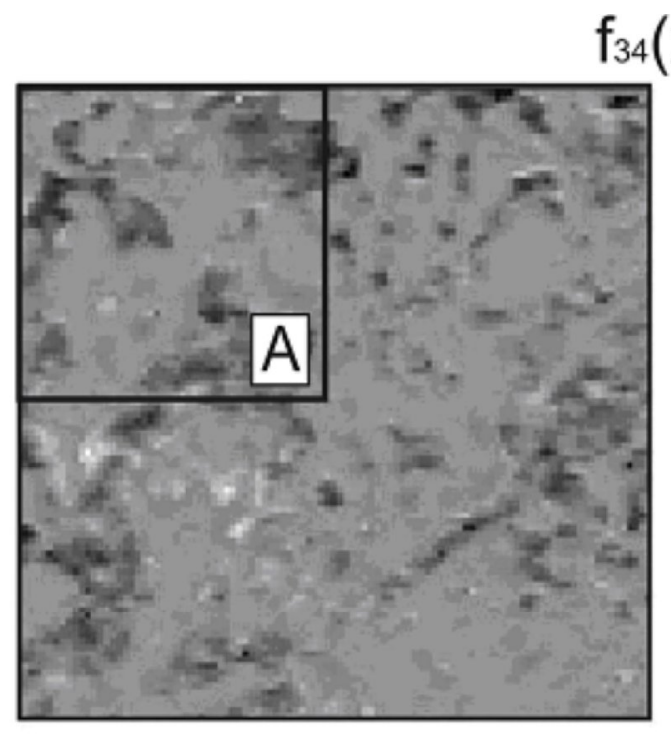

(a)

\section{$f_{34}(\mathrm{x}, \mathrm{y})$}

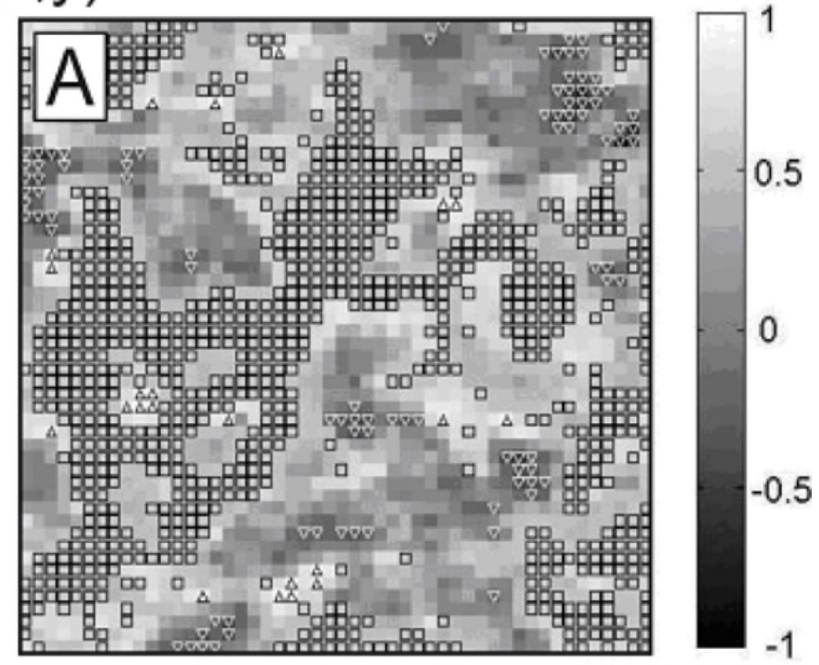

(b)

Fig. 9. Coordinate structure of the matrix element $f_{34}$ for myometrium (a). A - coordinate distribution of singular values (b) S-points $\left(f_{34}=1\right)$ labeled as $(\square),+$ C-points $\left(f_{34}=+1\right)-(\Delta)$ and -C-points $\left(f_{34}=-1\right)-(\nabla)$.

anisotropy of BT properties is basic in formation of singular structures (see distributions of S- and C-points) of the object boundary field and can be used in a further analysis of their interferential transformations.

\section{Statistic singular structure of the Mueller matrixes for biological tissues}

Figs 6 to 11 show statistical coordinate distributions for all the types of singularities inherent to the ensemble of matrix elements represented in Table 1 that were determined over the whole area of the sample under study.

As seen from these experimental data, the coordinate distributions of all the Mueller matrix elements for myometrium possess a developed network of singular values. Being based on this fact, we have offered singular differentiation of changes in the distribution of optical axis orientations in biological crystals that form the architectonic network, by using as an example the woman matrix tissue. 


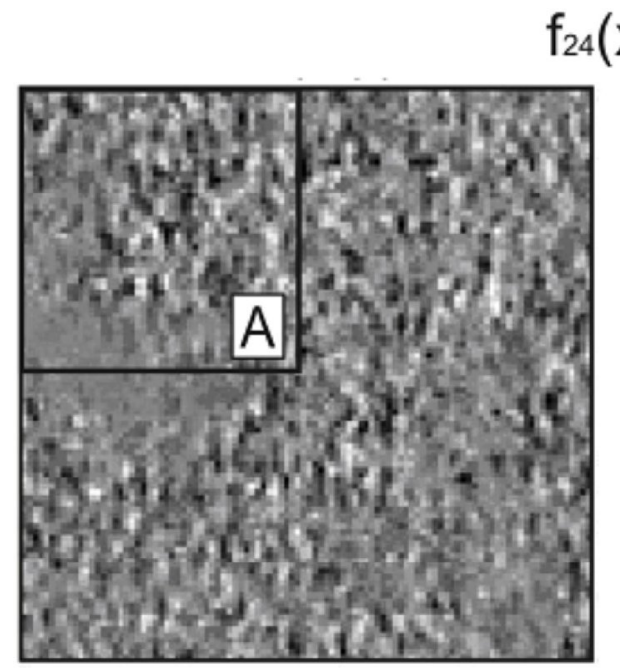

(a) $f_{24}(x, y)$

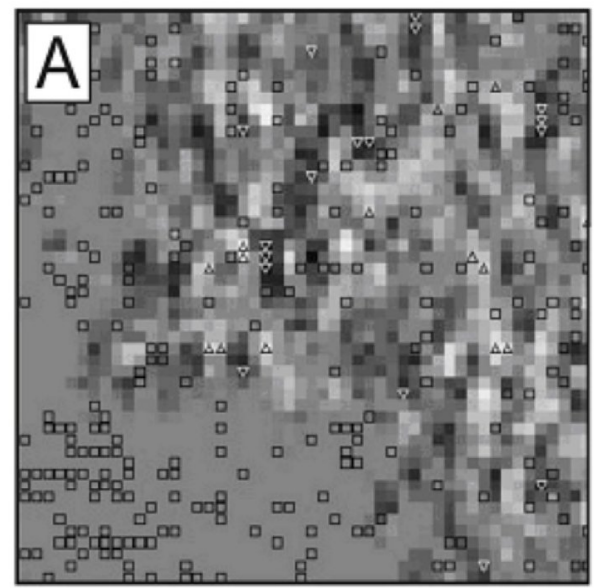

(b)

Fig. 10. Coordinate structure of the matrix element $f_{24}$ for myometrium (a). A - coordinate distribution of singular values (b) S-points $\left(f_{24}=1\right)$ labeled as $(\square),+$ C-points $\left(f_{24}=+1\right)-(\Delta)$ and -C-points $\left(f_{24}=-1\right)-(\nabla)$.

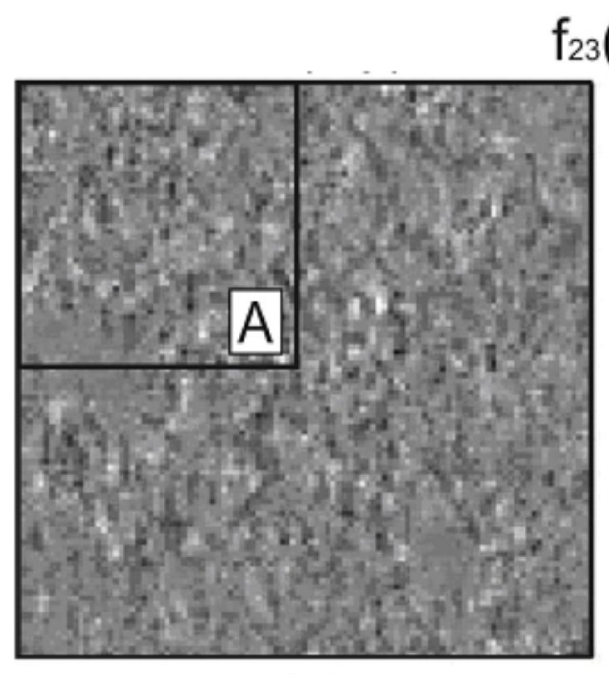

(a) $\mathrm{f}_{23}(\mathrm{x}, \mathrm{y})$

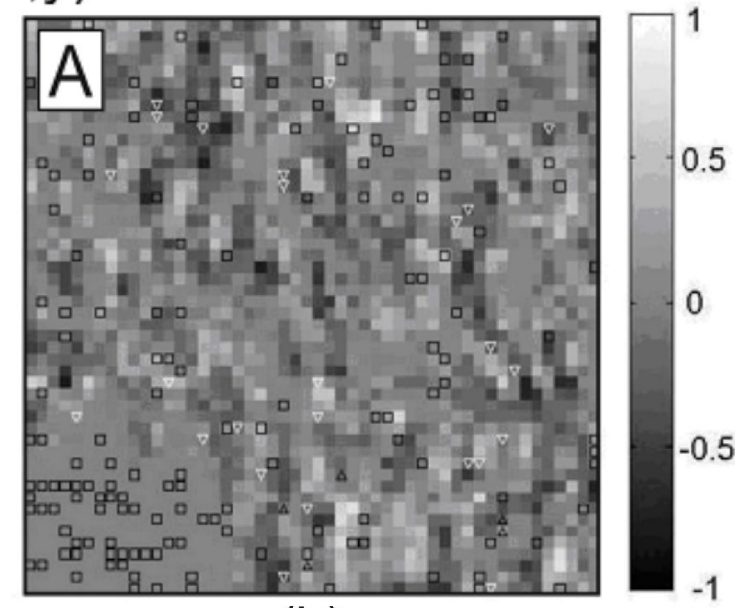

(b)

Fig. 11. Coordinate structure of the matrix element $f_{23}$ for myometrium (a). A - coordinate distribution of singular values (b) S-points $\left(f_{23}=1\right)$ labeled as $(\square),+$ C-points $\left(f_{23}=+1\right)-(\Delta)$ and - C-points $\left(f_{23}=-1\right)-(\nabla)$.

6. Mueller-matrix diagnostics of orientation changes of organic crystals in biological tissues

As objects for our experimental investigations, we used mounts of myometrium tissue of two types:

- biopsy of healthy tissue from a woman matrix (type A);

- biopsy of conditionally normal tissue from the vicinity of a benign hysteromyoma (type B).

Fig. 12 shows MMI of the element $f_{44}$ for myometrium samples of A and B types.

From the optical viewpoint, the obtained twodimensional distributions $f_{44}(x, y)$ characterize the degree of anisotropy in the matter of studied samples. Thereof, it can be easily seen that the birefringency value of the samples A and B is practically identical. It is confirmed by the close level of relative values for the matrix element $f_{44}(x, y)$ describing the tissues of $\mathrm{A}$ and B types $\left(f_{44}^{E}(x, y) \approx f_{44}^{A}(x, y)\right)$. In parallel with it, one can observe ordering the directions of optical axes inherent to anisotropical structures of type B myometrium.

Thus, the main parameter allowing differentiation of optical properties for the samples of this type is the orientation structure of their birefringent networks. 


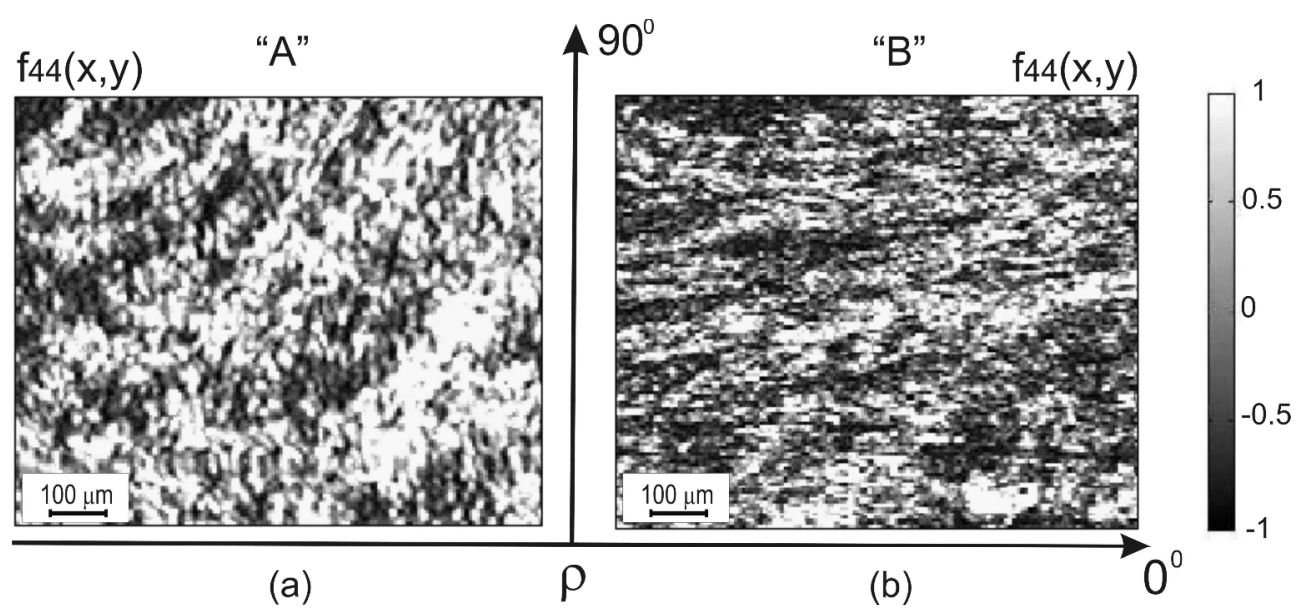

Fig. 12. MMI of the element $f_{44}$ for myometrium tissue of A (a) and B (b) types.

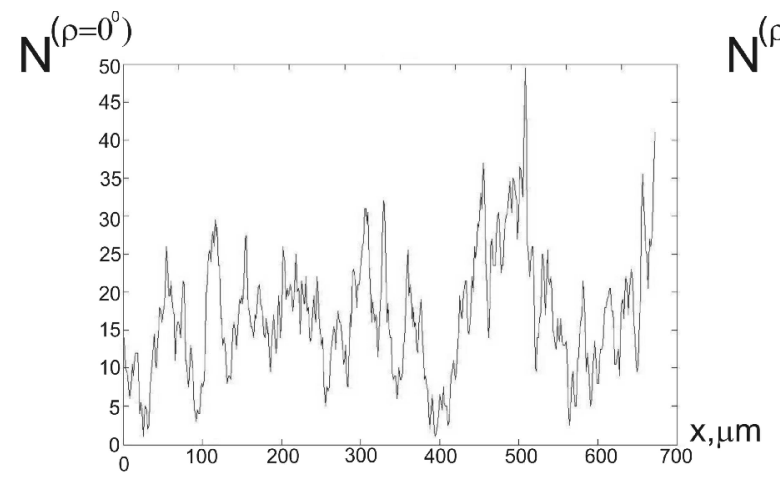

(a)

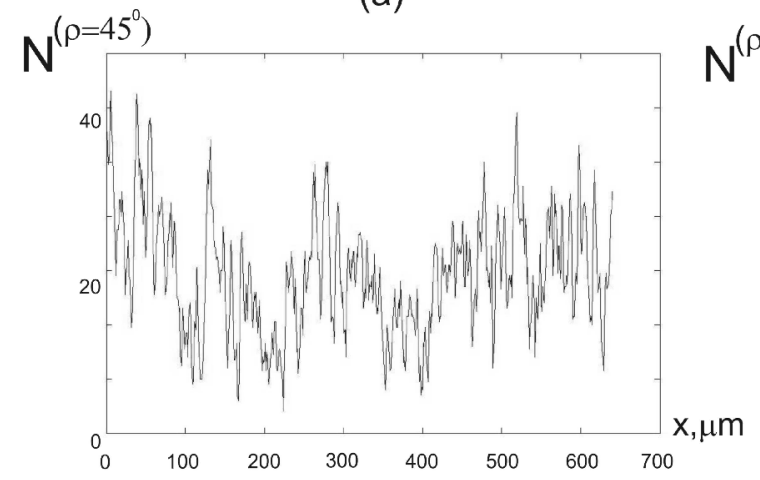

(c)
$N^{(\rho=}$

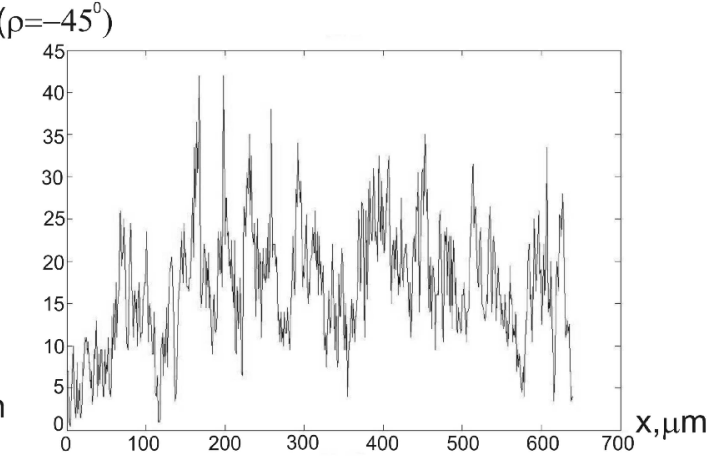

(d)

Fig. 13. Dependences of singularity numbers $N\left(\rho=0^{\circ}\right)$ (a), $N\left(\rho=90^{\circ}\right)(b), N\left(\rho=45^{\circ}\right)$

(c), $N\left(\rho=-45^{\circ}\right)$ (d) for myometrium tissue of A type.

To obtain objective criteria for Mueller-matrix singular differentiation of optical properties inherent to the myometrium samples of A and B types, we have used the following approach:

- measured in sequence were the MMI elements $f_{24,42}$ and $f_{34,43}$, that are basic to determine singular states $( \pm 1)$ formed in organic crystals with orthogonal orientations of optical axes $\left(\rho=0,90^{\circ} \rightarrow f_{34,43}= \pm 1\right.$; $\rho=+45^{\circ},-45^{\circ} \rightarrow f_{24,42}= \pm 1$ (Table 1);

- determined were graticubes for distribution of the singular values $f_{34,43}(x, y)= \pm 1$ and $f_{24,42}(x, y)= \pm 1$;

- determined was the set of dependences for the number of points corresponding to singular states MMI $N\left(\rho=0^{\circ}\right), N\left(\rho=90^{\circ}\right), N\left(\rho=45^{\circ}\right), N\left(\rho=-45^{\circ}\right)$;

- $N\left(\rho_{i}\right)$ dependences were processed using the following algorithms:

$$
\left\{\begin{array}{c}
Z_{M}^{\left(f_{34,43}\right)}=\frac{M\left(\rho=0^{\circ}\right)-M\left(\rho=90^{\circ}\right)}{M\left(\rho=0^{\circ}\right)+M\left(\rho=90^{\circ}\right)} ; \\
Z_{M}^{\left(f_{24,42}\right)}=\frac{M\left(\rho=45^{\circ}\right)-M\left(\rho=-45^{\circ}\right)}{M\left(\rho=45^{\circ}\right)+M\left(\rho=-45^{\circ}\right)} .
\end{array}\right.
$$

\section{(C) 2009, V. Lashkaryov Institute of Semiconductor Physics, National Academy of Sciences of Ukraine}



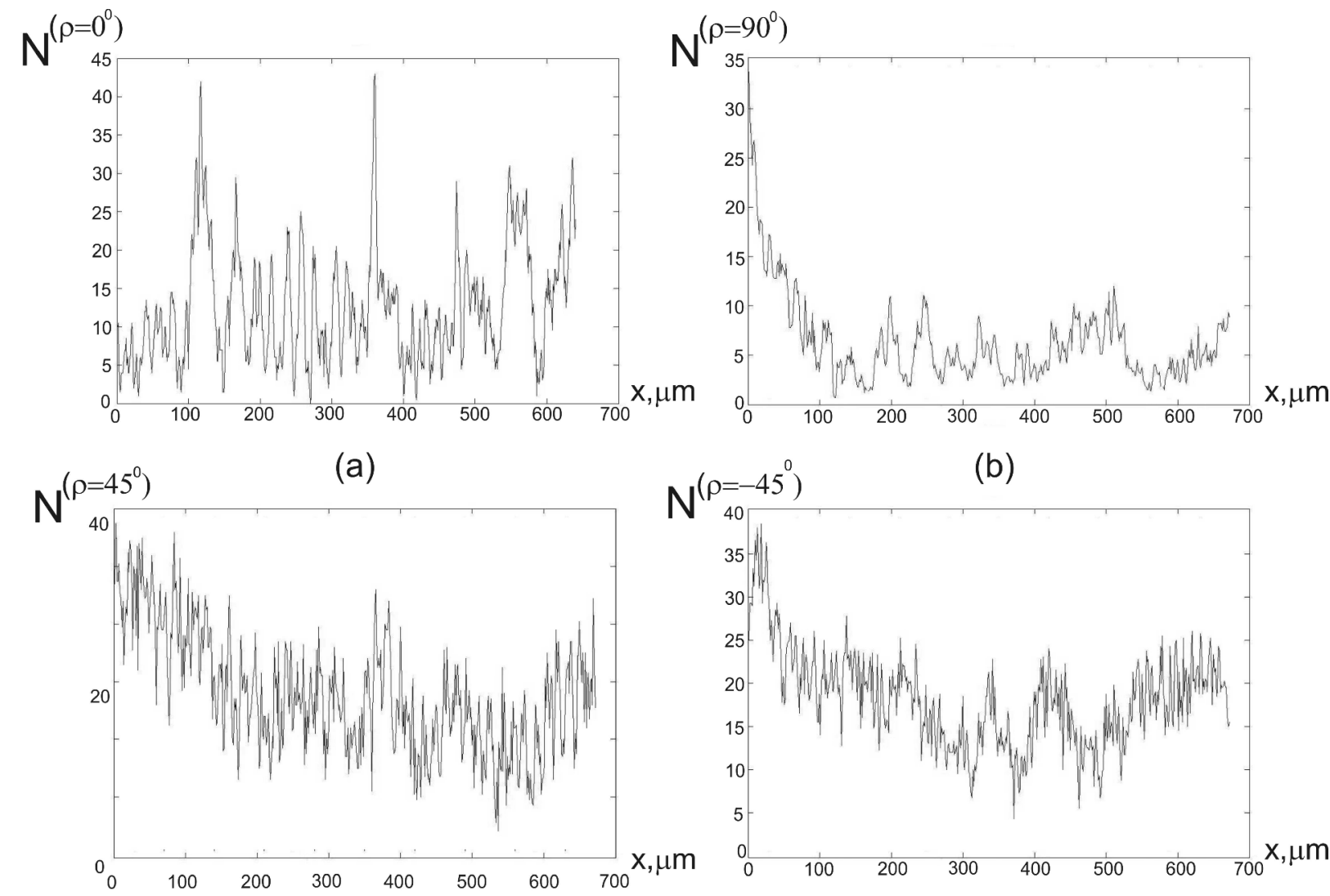

(c)

(d)

Fig. 14. Dependences of singularity numbers $N\left(\rho=0^{\circ}\right)$

(a), $N\left(\rho=90^{\circ}\right)$

(b), $N\left(\rho=45^{\circ}\right)$

(c), $N\left(\rho=-45^{\circ}\right)$

(d) for myometrium tissue of B type.

$$
\left\{\begin{array}{c}
Z_{\sigma}^{\left(f_{34,43}\right)}=\frac{\sigma\left(\rho=0^{\circ}\right)-\sigma\left(\rho=90^{\circ}\right)}{\sigma\left(\rho=0^{\circ}\right)+\sigma\left(\rho=90^{\circ}\right)} ; \\
Z_{\sigma}^{\left(f_{24,42}\right)}=\frac{\sigma\left(\rho=45^{\circ}\right)-\sigma\left(\rho=-45^{\circ}\right)}{\sigma\left(\rho=45^{\circ}\right)+\sigma\left(\rho=-45^{\circ}\right)} .
\end{array}\right.
$$

Here, $M\left(\rho_{i}\right)$ and $\sigma\left(\rho_{i}\right)$ are the average and dispersion of $N\left(\rho_{i}\right)$ distributions.

Shown in Figs 13 and 14 are the distributions of singularity numbers $N^{(j)}(x)$ for the myometrium tissue of A and B types.

In the case of myometrium tissue with pathological changes, one can observe asymmetry between the ranges of changes in values of the dependences $N(\rho)=0$ (Fig. 14, a) and $N(\rho)=90$ (Fig. 14, b).

The above results can be explained as based on the found relation between conditions providing formation of MMI singular values and orientation-phase structure of biological crystals in the myometrium tissue (Table 1).

Orientation structure of MMI for the element $f_{44}$ describing the myometrium tissue of $\mathrm{B}$ type
(Fig. 12, b) contains singular points $f_{44}(x, y)=1$ asymmetrically located in the direction $\rho=90^{\circ}$. Thereof, one should expect a maximal number of singular values for the element $f_{34}(x, y)=-1$ as compared to that of singular values $f_{34,43}(x, y)=1$ and $f_{24,42}(x, y)= \pm 1$.

Statistically found asymmetry in distributions of singular states for MMI describing the myometrium tissue of both types was estimated using the coefficients of asymmetry (15) and (16) introduced by us. Table 2 shows statistically averaged values of the coefficients $Z_{M}$ and $Z_{\sigma}$ within two groups of myometrium samples of $\mathrm{A}$ and $\mathrm{B}$ types.

Analysis of data represented in Table 2 allowed us to conclude:

- first- and second-order statistical moments for distributions of singular values $f_{34,43}(x, y)$ and $f_{24,42}(x, y)$ characterizing MMI of healthy myometrium tissue do not practically differ from zero, which is indicative of their azimuthal symmetry; 
Table 2. Asymmetry coefficients of Mueller-matrix singularities

\begin{tabular}{|c|c|c|}
\hline$Z$ & $\begin{array}{c}\text { Myometrium } \\
\text { (normal state) } \\
(25 \text { samples })\end{array}$ & $\begin{array}{c}\text { Myometrium } \\
\text { (pathological state) } \\
(23 \text { samples })\end{array}$ \\
\hline$Z_{M}^{f_{43,34}}$ & $0.03 \pm 0.005$ & $0.45 \pm 0.063$ \\
\hline$Z_{\sigma}^{f_{43,34}}$ & $0.02 \pm 0.004$ & $0.12 \pm 0.019$ \\
\hline$Z_{M}^{f_{42,24}}$ & $0.025 \pm 0.0036$ & $0.28 \pm 0.037$ \\
\hline$Z_{\sigma}^{f_{42,24}}$ & $0.03 \pm 0.0047$ & $0.14 \pm 0.021$ \\
\hline
\end{tabular}

- values of the asymmetry coefficient for distributions of MMI singular values $f_{34,43}(x, y)$ and $f_{24,42}(x, y)$ describing the pathologically changed myometrium tissue of B type grow practically by one order, which indicates formation of their azimuthal asymmetry related with the direction of pathological growth of birefringent protein fibrils.

Thus, the above analysis of statistical distributions describing the number of points for MMI singular values inherent to the set of elements $f_{i k}$ characterizing biological tissues of different kinds seems to be efficient in differentiation of phase and orientation changes in the structure of their birefringent components, which are related with changes in their physiological state.

\section{References}

1. S.C. Cowin. How is a tissue built? // J. Biomed. Eng. 122, p. 553-568 (2000).

2. O.V. Angelsky, A.G. Ushenko, Yu.A. Ushenko, Ye.G. Ushenko, Yu.Ya. Tomka, V.P. Pishak, Polarization-correlation mapping of biological tissue coherent images // J. Biomed. Opt. 10, No. 6, 064025 (2005).

3. J.F. de Boer and T.E. Milner, Review of polarization sensitive optical coherence tomography and Stokes vector determination // J. Biomed. Opt. 7, p. 359-371 (2002).

4. J.F. de Boer, T.E. Milner and J.S. Nelson, Two dimensional birefringence imaging in biological tissue using phase and polarization sensitive optical coherence tomography // In: Trends in Optics and Photonics (TOPS): Advances in Optical Imaging and Photon Migration. OSA, Washington, DC, 1998.

5. M.J. Everett, K. Shoenenberger, B.W. Colston and L.B. Da Silva. Birefringence characterization of biological tissue by use of optical coherence tomography // Opt. Lett. 23, p. 228-230 (1998).

6. A.G. Ushenko, D.N. Burkovets, Wavelet-analysis of two-dimensional birefringence images of architectonics in biotissues for the diagnostics of pathological changes // J. Biomed. Opt. 9, No. 4, p. $1023-1028$ (2004).
7. O.V. Angelsky, Yu.Y. Tomka, A.G. Ushenko, Ye.G. Ushenko, S.B. Yermolenko, Yu.A. Ushenko, 2-D tomography of biotissue images in pre-clinic diagnostics of their pre-cancer states // Proc. SPIE 5972, p. 158-162 (2005).

8. O.V. Angelsky, A.G. Ushenko, D.N. Burcovets, Yu.A. Ushenko, Polarization visualization and selection of biotissue image two-layer scattering medium // J. Biomed. Opt. 10, No. 1, 014010 (2005).

9. O.V. Angelsky, Yu.Ya. Tomka, A.G. Ushenko, Ye.G. Ushenko and Yu.A. Ushenko, Investigation of 2D Mueller matrix structure of biological tissues for pre-clinical diagnostics of their pathological states // J. Phys. D: Appl. Phys. 38, p. 4227-4235 (2005).

10. Shuliang Jiao, Wurong Yu, George Stoica, Lihong V. Wang, Optical-fiber-based Mueller optical coherence tomography // Opt. Lett. 28, p. 12061208 (2003).

11. Yu.A. Ushenko, Statistical structure of polarization-inhomogeneous images of biotissues with different morphological structures // Ukrainian Journal of Physical Optics 6, No. 2, p. 63-70 (2005).

12. O.V. Angelsky, A.G. Ushenko, Yu.A. Ushenko, Ye.G. Ushenko, Yu.Ya. Tomka, V.P. Pishak, Polarization-correlation mapping of biological tissue coherent images // J. Biomed. Opt. 10, No. 6, 064025 (2005).

13. O.V. Angelsky, A.G. Ushenko, and Yu.A. Ushenko, Polarization reconstruction of orientation structure of biological tissues birefringent architectonic nets by using their Mueller-matrix speckle-images // J. Holography Speckle 2, p. 7279 (2005).

14. A.G. Ushenko, and V.P. Pishak, Laser Polarimetry of Biological Tissue. Principles and Applications, In: Coherent-Domain Optical Methods. Biomedical Diagnostics, Environmental and Material Science (V.Tuchin, ed.). Kluwer Academic Publishers, 2004, p. 67-93.

15. J.F. Nye, Natural Focusing and the Fine Structure of Light. Institute of Physics Publishing, Bristol, 1999.

16. J.F. Nye, Polarization effects in the diffraction of electromagnetic waves: the role of disclinations // Proc. R. Soc. A 387, p. 105-132 (1983).

17. I. Freund, Bichromatic optical Lissajous fields // Opt. Communs. 226, p. 351-376 (2003).

18. J.F. Nye and J.V. Hajnal, The wave structure of monochromatic electromagnetic radiation // Proc. R. Soc. A 409, p. 21-36 (1987).

19. M.V. Berry and M.R. Dennis, Polarization singularities in isotropic random vector waves // Proc. R. Soc. A 457, p. 141-155 (2001).

20. A.D. Dolgov, A.G. Doroshkevich, D.I. Novikov and I.D. Novikov, Classification of singular points in the polarization of the cosmic microwave 
background and eigenvectors of the Stokes matrix // JETP Lett. 69, p. 427-433 (1999).

21. A.I. Konukhov and L.A. Melnikov, Optical vortices in a vector fields: the general definition based on the analogy with topological solitons in a 2D ferromagnet, and examples from the polarization transverse patterns in a laser // J. Opt. B: Quantum Semiclass. Opt. 3, S139-S144 (2001).

22. I. Freund, Poincare vortices // Opt. Lett. 26, p. 1996-1998 (2001).

23. J.F. Nye, Lines of circular polarization in electromagnetic wave fields // Proc. R. Soc. A 389, p. $279-290$ (1983).
24. I. Freund, Coherency matrix description of optical polarization singularities // J. Opt. A.: Pure and Appl. Opt. 6, S229-S234 (2004).

25. O.V. Angelsky, A.G. Ushenko and Ye.G. Ushenko, 2-D Stokes polarimetry of biospeckle tissues images in pre-clinic diagnostics of their pre-cancer states // J. Holography Speckle 2(1), p. 26-33 (2005).

25. O.V. Angelsky, A.G. Ushenko, Yu.A. Ushenko and Ye.G. Ushenko, Polarization singularities of the object field of skin surface // J. Phys. D: Appl. Phys. 39, p. 3547-3558 (2006).

26. O V Angelsky, A.G. Ushenko, Ye.G. Ushenko and Y.Y. Tomka, Polarization singularities of the biological tissue images // J. Biomed. Opt. 11, No. 5, 054030 (2006). 